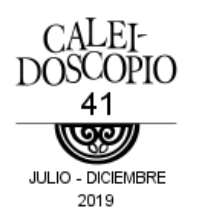

DOSCOPIO

41

2019

\title{
El proceso: elemento fundamental en la investigación sociocultural. Apuestas desde América Latina
}

The process: fundamental element in sociocultural research. Ventures from Latin

America

GABRIELA ITZAGUERI MENDOZA SÁNCHEZ

Universidad Autónoma de Aguascalientes, México

\section{RESUMEN}

El presente texto trata de colaborar con las propuestas de autores como Alfonso Torres Carillo y Hugo Zemelman para los planteamientos teórico-metodológicos, con el llamado a la resignificación desde instancias subalternas. Teniendo como punto de emisión a América Latina en las investigaciones sociales y culturales, se pone énfasis en el proceso como elemento fundamental que involucra a experiencias desde el arte.

Palabras clave: sociocultural; proceso; América Latina; metodología.

\section{ABSTRACT}

The present text wishes to contribute with approaches from authors such as Alfonso Torres Carrillo and Hugo Zemelman in theory and methodology, convening to the 
resignification from subaltern instances. Having Latin America as an emission point in social and cultural research, the process is emphasized as a fundamental element that involves experiences comimg from art.

Keywords: social and cultural studies; process; Latin America; methodology.

Desde la perspectiva de distintos autores, se ha visto que la investigación sociocultural disuelve sus fronteras con otras disciplinas, no únicamente de las ciencias sociales, sino también tomando elementos de diversas áreas, para tratar de acercarse a la comprensión de las realidades cambiantes.

Es decir, ha sido producto de la realidad social, el que las ciencias sociales han tenido que abrirse. Ante esta autocrítica de las ciencias sociales, trataré de incorporar en el último momento de este texto, una visión igualmente crítica, de la historia del arte y de la filosofía del arte.

Cuando los estudios socioculturales centran la atención en el proceso, por un lado, fijan el objetivo de las disciplinas sociales en la búsqueda, en la investigación; aunque la expresión parece reiterativa, trata de ser explicita. Por otro lado, a partir de poner mayor interés en el proceso, las metodologías y técnicas se especifican y se detallan en cada trabajo, incorporando con ello una dimensión didáctica, aunque, sabemos que cada investigación tendrá que crear sus propuestas metodológicas.

Como ejemplo, también de esta transformación, está el arte, aunque no de manera lineal, ni determinada para 
toda la geografía; se fue fijando el interés en los procesos del arte. Las técnicas que anteriormente se llegaron a considerar como secretos del "genio" artístico, fueron compartidas de manera más amplia en las Escuelas de arte. En la actualidad, las técnicas por novedosas que sean, se muestran al público, y las bitácoras de artista, los cuadernos de trabajo y cualquier otro producto del proceso de "creación" se exhiben en diferentes salas, es decir, han sido puestas en valor.

Estas facetas, que antes eran un misterio acerca del trabajo de los artistas plásticos, formaban parte de un momento previo a la obra que podía exhibirse, podríamos atrevernos a decir, que estaban en la situación liminal. Alfonso Torres Carrillo, ha denominado la "investigación social no canónica generada en los bordes de las ciencias sociales, como investigación desde el margen o liminal"1 y apuesta por la incorporación de conceptos, métodos y técnicas de la literatura, el cine, de los saberes ancestrales, por supuesto, recreándolos y articulándolos 2 . Reconocemos que los temas provenientes de las áreas mencionadas, sí han estado presentes en las ciencias sociales.

La propuesta de denominación de Torres Carrillo, tiene sinónimos con las de otros autores desde América Latina, e incluso desde otras latitudes, al margen de la tradición de Europa occidental, Torres para explicar la fase o a los personajes liminales, lo asimila con:

El transeúnte ritual no tiene nada, ni estatuto, ni propiedad, ni signos, ni rango que lo distingan de quienes comparten su situación. Actualmente, esta condición de transeúntes, de monstruos del umbral, la desempeñan

${ }^{1}$ Torres Carillo, Alfonso. "Investigar en los márgenes de las ciencias sociales", en http://www.ucentral.edu.co., p.54.

${ }^{2}$ Torres Carrillo, Alfonso. "Investigar en...", p. 52.

$$
\text { C A L E I D O S C C O P I }
$$


personajes como los inmigrantes, los adolescentes, los enamorados, los artistas y los outsider en general ${ }^{3}$.

Se podría, además de lo evidente en la propuesta de Torres, considerar la descripción anterior, como una invitación a quienes están en esta situación para proponer perspectivas y técnicas más creativas y quizá con mayor posibilidad de diferenciarse de los medios ya muy institucionalizados para estudiar los fenómenos socioculturales.

Esta propuesta de Alfonso Torres, que se suma a autores que él mismo incorpora a su argumentación, como Hugo Zemelman, no pierde de vista la tradición occidental, se trata de una respuesta crítica a una tradición científica. Cabe señalar que esta tradición se ha ido ampliando poco a poco, y podríamos identificar un momento previo a través de la historia del arte.

Esta preocupación de largo aliento, dentro de la Historia del arte, la podemos reconocer con Jacques Thuillier-por cierto, hoy considerado como un conservador-, quien nos dice, que los primeros museos de arte y sus círculos de críticos y coleccionistas incorporaban únicamente piezas que correspondían a sus propias naciones, o en su defecto, mostraban objetos pertenecientes a otras latitudes pero que, de igual manera correspondieran al espíritu nacionalista del país en cuestión. Según este teórico, Francia fue el primer país en mostrar interés en el arte de otros lugares ${ }^{4}$.

Para este proceso de apertura, la sociología y la antropología, han ido mostrando la existencia de varias culturas, de varias realidades sociales; y los estudiosos

${ }^{3}$ Delgado, 1999., quien retoma a Victor Turner, citado en Torres Carrillo, Alfonso., “Investigar en...", p. 58.

4 Thuillier, Jacques. Teoría General de la Historia del Arte., Fondo de Cultura Económica, México, 2006. 
desde América Latina, van pugnando por tener una postura política como investigadores, hecho que no surge de manera gratuita. Siguiendo a Torres, nos dice que han sido creadores de perspectivas de borde, de razonamientos de umbral, producto a la vez, de una historicidad singular de las sociedades latinoamericanas ${ }^{6}$, de sus problemáticas. Entonces, el investigador ante estas situaciones se ha visto más comprometido, nos señala Torres:

El punto de partida de una investigación temática, de una investigación participativa, de una recuperación de memoria colectiva o de una sistematización de experiencias es el tener claridad acerca de las preguntas políticas sobre el porqué, para qué y para quiénes va a tener sentido la investigación a realizar, pregunta muchas veces ausente en los proyectos convencionales ${ }^{7}$.

Así desde esta afirmación metodológica, Torres expresa que el lugar de la definición de las preguntas, categorías y perspectivas interpretativas es frente al contexto y no ceñidos a los parámetros de una disciplina o teoría predeterminada ${ }^{8}$. Entonces, fijando la atención en el proceso, la investigación distará de ser una comprobación -quizá forzada- de una teoría.

Las prácticas investigativas marginales buscan relacionarse con lo teórico no desde una lógica deductiva o demostrativa, sino como "caja de herramientas". Se acude a enfoques y conceptos provenientes de diferentes

${ }^{5}$ Torres Carrillo, Alfonso., "Investigar en...", p.56.

${ }^{6}$ Torres Carrillo, Alfonso., "Investigar en...", p.54.

7 Torres Carrillo, Alfonso., "Investigar en...", p.57.

${ }^{8}$ Torres Carrillo, Alfonso., "Investigar en...", y Zemelman, Hugo., Pensar teórico y pensar epistémico: Los retos de las ciencias sociales latinoamericanas., en Instituto Pensamiento y Cultura en América, A. C. 
disciplinas, [..] resignificados en función de la especificidad de los problemas de investigación?.

Podríamos decir, que desde esta tendencia latinoamericana, asumida como una postura, donde el pensamiento debe entenderse como una postura, -dice Hugo Zemelman-, más específicamente, "la centralidad del pensamiento epistémico es la pregunta, no el predicado, no es la atribución de propiedades." ${ }^{\text {"0 }}$ ¿Por qué es necesario hacer adecuaciones teóricas y metodológicas en la investigación? Zemelman nos ayuda a responder, al reflexionar acerca del desajuste, el desfase que existe entre la realidad y la teoría", la realidad social es móvil, cambiante, y la teoría se va modificando de acuerdo a las necesidades sociales. Sin embargo, para Zemelman desde las Universidades latinoamericanas hace falta asumir compromisos conceptuales, atreverse a proponer revisiones teóricas y podemos destacar, con este autor, "la necesidad de una constante resignificación" de los conceptos $^{12}$. El mismo Zemelman, retoma a Bachelard, para advertir, que si "La tarea de la ciencia es poner nombre a las cosas, se corre el riesgo de poner nombre viejo a cosas nuevas o creer que porque no tienen nombre, en el momento que se plantea, son innombrables."13

Ante la realidad social, producto del fenómeno de interiorización de hábitos, asumimos nuestra realidad

\footnotetext{
9 Torres Carrillo, Alfonso., “Investigar en...”, p. 59.

${ }^{10}$ Zemelman, Hugo., Pensar teórico..., Con esta afirmación, coloca el pensamiento epistémico en la discusión clásica con Karl Popper., p. 4.

${ }^{11}$ Zemelman, Hugo., Pensar teórico..., p.1.

${ }^{12}$ Zemelman, Hugo., Pensar teórico..., p.1.

13 Bachelard, La Racionalidad Científica y La Filosofía del No, citado por Zemelman, Hugo., Pensar teórico..., p.5.
} 
como: La realidad ${ }^{14}$, entonces, es quizá más complejo distinguir cuáles transformaciones necesitan nuevas denominaciones, o ampliar el sentido de las que las signan. En el "mundo del arte", tal vez por tratarse de una ruptura con la cotidianidad, se vuelve más clara la necesidad de que surjan nuevos conceptos y cambios teóricos importantes, para poder explicar las manifestaciones artísticas que van respondiendo a los diferentes momentos históricos, es por ésto que, nos sobran ejemplos de las resignificaciones dentro del arte, y sin embargo, aun en estas áreas, se presenta comúnmente el hecho de poner nombres viejos a cosas nuevas, y el que las categorías preconcebidas se utilicen sin mucha reflexión.

Pero, sí tenemos buenos ejemplos desde las ciencias sociales y las humanidades, particularmente en la contemporaneidad, de la resignificación de conceptos, se ha dado, por ejemplo, el movimiento de dichos conceptos de disciplinas de las ciencias naturales hacia la filosofía o la antropología.

Podríamos atrevernos a señalar que ha tenido que ver también, con la necesidad de construir identidades desde América Latina, o desde la diáspora, y de ahí sus teóricos. Sobre todo: la necesidad de resignificar y las propuestas más vitales, parecen surgir, no únicamente en los sitios de conflictos sociales, sino en los espacios intermedios ${ }^{15}$, liminales ${ }^{16}$, donde se han construido nuevas identidades ${ }^{17}$.

\footnotetext{
${ }^{14}$ Los planteamientos en la Fenomenología social, con Berger y Luckmann.

${ }^{15}$ El espacio propuesto por Homi Bhabha. El lugar de la cultura. Existe una publicación con la traducción de César Aira.

${ }^{16}$ La denominación resignificada por Torres Carrillo, Alfonso., "Investigar en...

${ }^{17}$ El estudio que comprende el reciente libro del historiador Víctor M. González Esparza, titulado Resignificar el mestizaje. Tierra Adentro. Aguascalientes, Nueva Galicia, siglo XVII y XVIII. Universidad Autónoma de Aguascalientes y El Colegio de
} 
Volvamos con Zemelman a comprender la investigación como un proceso, porque tiene "como requisito elemental construir el conocimiento de aquello que no se conoce, no de aquello que se conoce."18 ¿Para qué plantearnos una investigación sobre aquello que ya conocemos?

El situarnos ante algo por descubrir, que tendremos que abordar con conocimiento de su contexto, es decir, de su historia, de su tradición, y producto de determinadas transformaciones, que habremos de tratar de comprender y explicar. Entonces, necesitamos comprometer nuestro pensamiento epistémico como "el esfuerzo del hombre por construir una relación con la realidad."19

Investigadores como Zemelman o Néstor García Canclini, nos persuaden no únicamente por su discurso, sino por "su capacidad de desplegar energías transformadoras, ${ }^{20}$ en el primero, se distingue una característica que Torres identifica en los investigadores marginales, que llevan su compromiso con las problemáticas sociales al grado de hacer activismo ${ }^{21}$. Un compromiso más que se puede identificar en Zemelman, es la creación del Instituto Pensamiento y Cultura en América Latina. Este gesto es una afirmación de existencia frente al pensamiento occidental, sin salirse, sino expandiendo sus límites. En el segundo, siguiendo su trayectoria podemos ver sus propuestas conceptuales, su fusión de metodologías cuantitativas con cualitativas para revalorar dinámicas culturales particulares, insertar

\footnotetext{
San Luis, A. C. México, 2018.

${ }^{18}$ Zemelman, Hugo., Pensar teórico..., p. 10.

${ }^{19}$ Zemelman, Hugo., Pensar teórico..., p. 8.

${ }^{20}$ Torres Carrillo, Alfonso., "Investigar en..., p. 61.

${ }^{21}$ Torres Carrillo, Alfonso., "Investigar en..." p.p. 59-60.
}

100 NÚM. 41 JULIO - DICIEMBRE 2019 doi:10.33064/41crscsh1841 
preguntas nuevas ante las transformaciones sociales, por ejemplo, hacia las aspiraciones de los jóvenes en la actualidad $^{22}$.

Sin embargo, no quiere decir que, para nuestras prácticas dentro de la investigación sociocultural, nos vaya a ser sencillo el camino de distanciarnos de los prejuicios que nos han constituido y de los que podríamos no estar muy conscientes porque los tenemos completamente interiorizados, asimilados. Construir el andamiaje que nos permita tener un pie dentro de la realidad, a la que en cierto sentido pertenecemos y que queremos estudiar y otro pie fuera, lo suficiente para permitirnos una aproximación a esa realidad, sin pre-concepciones, será, en efecto, todo un esfuerzo.

Lo planteado en estos cambios metodológicos, nos advierte de que, si bien ha sido justificado en un sinfín de investigaciones una aplicación deductiva, más con tintes de verificación de una hipótesis, trabajar con la centralidad puesta en la teoría, también conlleva las pre-concepciones y la posible aplicación de ideologías, que no nos permitan encontrar los matices propios de una realidad compleja.

Zemelman, nos da herramientas para acercarnos a comprender la complejidad de lo real, al exigir se le estudie en varios recortes de la realidad, así como reconocer que sus temporalidades son múltiples y entender que los fenómenos son universos de significación, que tienen lugar a través de coyunturas que forman parte de tendencias a largo plazo y que están conformados por distintos contextos relacionados entre $\mathrm{Si}^{23}$. Atendiendo dichas exigencias, se hace manifiesta la

\footnotetext{
22 García Canclini, Néstor. Pronunció una conferencia que me pareció muy provocadora, actividad dentro de la celebración por un aniversario de la carrera de sociología en la U.A.A.

${ }^{23}$ Zemelman, Hugo., Pensar teórico..., p.p. 15-16.
} 
necesidad de valernos de metodologías de disciplinas distintas, y de ser creativos.

La crítica que desde los estudios socioculturales se hace a la especialidad en una disciplina, viene de ese reconocimiento a la necesidad de observar los distintos contextos que se relacionan con un fenómeno social. Para ejemplificar, los límites de la especialización disciplinar y para cerrar dando respuesta a la sugerencia que aparece al principio de este ensayo, veamos la historia del arte, donde podemos encontrar una gran cantidad de trabajos que describen los objetos artísticos; algunos más incorporan interpretaciones críticas, basadas en las teorías que se han realizado para explicar a través de los distintos lenguajes de las artes; pero, tal vez queda espacio para integrar en las investigaciones, un énfasis en las transformaciones sociales que están alrededor de los fenómenos artísticos.

En el caso de la filosofía del arte, me parece se podrían estrechar más las relaciones con la sociología y la antropología, lo que permitiría que hubiera mayor claridad acerca del proceso que va del surgimiento de una manifestación artística a la teorización sobre ésta, así como practicar de manera más constante una confrontación de la teoría con la realidad.

Un estudio que cuide los procesos, tendrá menos posibilidades de colocar conceptos inadecuadamente en la interpretación de la realidad que observa. En las tesis realizadas en los posgrados de arte, que en su mayoría son muy recientes, es fácil encontrar, una falta de atención a las transformaciones estéticas en la propuesta de cada artista y a cómo cada cual responde también de manera diferenciada a su "tiempo." De ahí que, frecuentemente vemos un predominio de las categorías de impresionismo o nacionalista. 
Así, se pueden considerar las propuestas de los autores que se han elegido para este ensayo, como ejes para pensar en las propuestas de investigación. Estas últimas líneas, sirven para apuntar la importancia de las argumentaciones que los estudiosos nos dan, para afinar la conciencia que tiene el investigador, acerca de que emite, no una verdad total sobre la realidad, sino una aproximación que no desconoce la subjetividad del que investiga ${ }^{24}$ y que sus concepciones son producto también, de la interacción con los demás.

\section{REFERENCIAS}

Orozco Guillermo y González Rodrigo. Una coartada metodológica. Abordajes cualitativos en la investigación en comunicación, medios y audiencias. Serie Brújula.

Productora de contenidos culturales. México, 2012. Thuillier, Jacques. Teoría General de la Historia del Arte., Fondo de Cultura Económica, México, 2006.

Torres Carillo, Alfonso. "Investigar en los márgenes de las

ciencias sociales", en http://www.ucentral.edu.co.

Zemelman, Hugo., Pensar teórico y pensar epistémico: Los retos de las ciencias sociales latinoamericanas., en Instituto

Pensamiento y Cultura en América, A. C.

${ }^{24}$ Se puede confrontar con el trabajo de Orozco Guillermo y González Rodrigo. Una coartada metodológica. Abordajes cualitativos en la investigación en comunicación, medios y audiencias. Serie Brújula. Productora de contenidos culturales. México, 2012. Y con: Flick, U., Introducción a la investigación cualitativa, Morata, España, 2012.

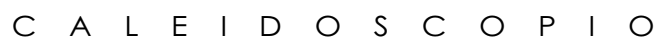


Gabriela Itzagueri Mendoza Sánchez (gabrielaitzagueri@yahoo.com.mx) es estudiante del Doctorado en Estudios Socioculturales, en la Universidad Autónoma de Aguascalientes. Es maestra en Arte Contemporáneo, licenciada en Artes Visuales y egresada de diseño gráfico. Ha trabajado en la docencia, la investigación y la creación artística (ORCID $\underline{\text { 0000-0003-3986-8897). }}$

Recibido: 23/03/2019 Aceptado: 14/05/2019

CÓMO CITAR ESTE ARTÍCULO

Mendoza Sánchez, G. I. (2019). El proceso: elemento fundamental en la investigación sociocultural. Apuestas desde América Latina. Caleidoscopio - Revista Semestral de Ciencias Sociales y Humanidades, 23(41), 93-104. doi:10.33064/41crscsh1841 